\title{
Allocation Mechanisms Without Reduction
}

\author{
David Dillenberger Uzi Segal*
}

February 25, 2021

\begin{abstract}
We study a simple variant of the house allocation problem (onesided matching). We demonstrate that agents with recursive preferences may systematically prefer one allocation mechanism to the other, even among mechanisms that are considered to be the same in standard models, in the sense that they induce the same probability distribution over successful matchings. Using this, we propose a new Priority Groups mechanism and provide conditions under which it is preferred to two popular mechanisms, Random Top Cycle and Random Serial Dictatorship.

JEL codes: C78, D81.

Key words: House allocation problem, Non-expected utility, Random Top Cycle, Random Serial Dictatorship, Reduction of compound lotteries.
\end{abstract}

\section{Motivation}

Many goods are allocated using randomizing devices. These include, among others, public schools, course schedules, or dormitory rooms to students, and shifts, offices, or tasks to workers. Standard analysis of allocation mechanisms is interested in their efficiency and in possible manipulations of their

*David Dillenberger, Department of Economics, University of Pennsylvania (ddill@sas.upenn.edu) and Uzi Segal, Department of Economics, Boston College (segalu@bc.edu). We thank Yoram Halevy, Tayfun Sönmez, Rani Spiegler, Utku Ünver, and Rakesh Vohra, as well as the editor and four anonymous referees, for their helpful suggestions. 
outcomes, but typically not in the way these procedures are perceived by the agents to whom outcomes are allocated. The literature on allocation problems, and more generally on one-sided and two-sided matching (for a survey, see Abdulkadiroğlu and Sönmez (2013)), typically maintains the assumption that agents are only interested in the overall probability they will receive their desired outcome. This assumption implies the equivalence of different randomized mechanisms (Abdulkadiroğlu and Sönmez (1998); see also Pathak and Sethuraman (2011)). Nevertheless, we show that taking into account the procedures that generate this probability may be important, and seemingly similar mechanisms like Random Top Cycle and Random Serial Dictatorship can be ranked differently when preferences over compound lotteries are taken into consideration. Moreover, we use this insight to propose a new mechanism which may be better than those currently discussed in the literature.

The basic structure we investigate is simple: $N$ units of two different types need to be allocated to $N$ individuals, one per person. For example, $N$ dorms - some face west and the other face east - that need to be allocated to incoming students. Some will prefer one type and some will prefer the other. Since there are only two types, there is no room for strategic manipulations and agents' optimal strategy is to reveal their true preferences. For tractability, in the formal analysis we will confine attention to the case of a large population: a continuum of agents and units.

We first take an ex-ante approach, where agents are yet to learn their own preferences (as well as those of the other agents) over the goods. These preferences are revealed after the first part of the procedure takes place. In professional sports, for example, teams typically know their rank in the draft before they know which positions they would like to fill. This will become clearer by the time they know the draft prospects and the medical condition of their current roster for next year. In a school context, prospective students often attend visit days and open houses long after the assignment procedure has been announced. Individuals in such situations thus view possible mechanisms as compound lotteries, that is, lotteries over the interim probabilities of receiving their desired outcome. Crucially, our analysis is based on the assumption that sequential probabilities are not taken by individuals to be the same as their product. In other words, individuals do not obey the reduction of compound lotteries axiom, according to which an agent should be indifferent between any multi-stage lottery and the single-stage lottery that induces the same probability distribution over final outcomes. 
Extensive experimental results suggest that individuals often fail to reduce compound lotteries to simple ones using probability laws (see, among others, Halevy (2007), Abdellaoui, Klibanoff, and Placido (2015), Harrison, Martinez-Correa, and Swarthout (2015), and Masatlioglu, Orhun, and Raymond (2017)). This is not necessarily a mistake, as is demonstrated by the following example. One hundred doses of vaccine are available for one hundred people. Two doses guarantee immunization, while one dose only reduces the probability of developing severe symptoms by half. You can either randomly select half the people and give each two doses, or give one dose to everyone. As a patient, are you indifferent between these two scenarios? More broadly, violations of the reduction axiom may reflect preferences over different processes even if they lead to the same overall distribution over final outcomes, or intrinsic preferences towards the timing of resolution of uncertainty.

Many theoretical models in which the reduction of compound lotteries axiom is relaxed have been proposed. We follow this approach and postulate that individuals have recursive preferences over compound lotteries. This structure allows us to distinguish between mechanisms that induce the same lotteries under the reduction assumption. ${ }^{1}$ A notable case is the two aforementioned mechanisms, Random Top Cycle (TC) and Random Serial Dictatorship (SD), which are no longer indifferent to each other without this assumption. We show conditions under which each of them is preferred to the other (see Section III). Moreover, it is this perception of mechanisms that enables us to propose a new Priority Groups (PG) mechanism, which under familiar conditions is considered better than both by the receiving agents.

The PG mechanism has the following structure. In the first stage, each individual is informed which of the two goods he is going to receive. In addition, he is informed to which 'trading group' he belongs, where being in a certain group determines the probability at which the individual will be able to trade, if desired, in the second stage. In the second stage each individual learns his preferences. Those who hold their desired good keep it. The remaining individuals can trade, where for those who hold the good for which there is an excess supply, the probability of being selected to trade is the one assigned to them in the first stage. Priority groups are common in many

\footnotetext{
${ }^{1}$ The mechanism design literature often ignores this sequential resolution of uncertainty. If a person submits his preferences to an algorithm and learns nothing until his final outcome is revealed, he probably will not view this as a compound lottery.
} 
industries, for example, in the airline industry. While priority groups often depend on some merit (e.g. the amount spent and miles flown in the previous calendar year), in the alternative we suggest the assignment to groups is random.

Intuitively, the different mechanisms we consider induce lotteries over interim probabilities that can be ranked in terms of "riskiness." Following Machina (1982), we approximate local behavior by expected utility functionals, and the curvatures of these local utility functions determine the desirability of a mechanism. For example, TC generates a less risky distribution than SD, and thus is preferred if all local utilities are concave. We outline conditions over these utilities that determine preferences over mechanisms and show that these conditions can also be linked to attitudes towards the timing of resolution of uncertainty (see section IV).

While our analysis is mainly focused on the ex-ante approach, it can also be applied to the interim case, in which each individual learns his place in the mechanism after he already knows his type. We demonstrate this in Section VI. Here again, the absence of the reduction assumption allows us to examine the performance of seemingly identical mechanisms, and to show conditions under which TC or SD is superior to the other and conditions where PG is preferred to both. More generally, it is important to emphasize that our analysis is not restricted to any specific order. It applies whenever mechanisms involve some sequential resolution of uncertainty (even with more than two stages) and individuals do not obey the reduction of compound lotteries assumption.

The rest of the paper is as follows: Section II introduces the basic structure and the TC and SD mechanisms. Section III describes the preferences we consider and compares TC to SD. Section IV discusses the PG mechanism and provides conditions under which it is preferred to both TC and SD. Section V extends our analysis beyond the class of preferences we studied in the previous sections. Section VI considers the interim case. Section VII concludes with some further discussion.

\section{Two Allocation Mechanisms}

Consider the following continuum variant of the house allocation problem (Hylland and Zeckhauser (1979)). There are goods of two types, $g_{1}$ and $g_{2}$ in proportion $p: 1-p$, to exactly supply the total quantity needed to ac- 
commodate a $[0,1]$ continuum of agents. All agents have the same stochastic preferences, where with probability $q$ each prefers $g_{1}$ to $g_{2}$ (independently of the preferences of others). We normalize payoffs so that the utility from the desired outcome is 1 and the utility from the other outcome is 0 . We analyze below the case of excess supply of $g_{1}$, that is, $p>q$. The analysis of the case $p<q$ is similar.

In this section we consider two familiar mechanisms, each consisting of two stages.

- Random Top Cycle (TC): In the first stage, the goods are randomly allocated among the agents, so that the probability of person $i$ holding good of type $g_{1}$ or $g_{2}$ is $p$ or $1-p$, respectively. In the second stage, the entire profile of preferences is revealed and trade, if needed, takes place. Those who like their holding will keep it. The rest will trade according to the following schedule: If $m$ proportion of people holding one type of good and $\ell<m$ proportion of people holding the other type are unhappy with their holding, then the latter group will trade and get their desired outcome, while $\ell$ out of the former group will be selected at random and get their preferred option. The other $m-\ell$ will keep their undesired outcome. ${ }^{2}$

- Random Serial Dictatorship (SD): In the first stage the order of the agents is randomly determined, so that the probability of each person being in the top $m$ part of the queue is $m$. In the second stage, the entire profile of preferences is revealed. The agents then choose goods according to the order determined in the first stage. Agents get their desired outcome if, when their turn arrives, such a unit is still available.

We first adopt an ex-ante perspective. In both cases, we assume here that in the first stage, before individuals know their preferences, the uncertainty of the relevant mechanism is revealed. In the second stage, people learn their preferences and act according to the outcome of the first stage. It is clear from the descriptions of the above mechanisms that they involve some sequential revelation of information: The outcome of the mechanism and the revelation of individual preferences. However, it is easy to see that both TC

\footnotetext{
${ }^{2}$ This is a variant of the classic top cycle mechanism. It can equivalently be formulated more closely to the familiar top cycle as a problem of matching with indifferences and using a specific tie-breaking rule. Since the environment we consider is simple, we maintain our formulation and slightly abuse the title "cycle."
} 
and SD lead to the same overall probability of success, and are hence deemed indifferent if agents are only interested in the overall probability at which they will receive their desired outcome (see Abdulkadiroğlu and Sönmez (1998)).

Suppose that the individual knows that he will face a binary lottery of the form $(x, \pi ; y, 1-\pi)$ where $x$ and $y$ are fixed and $x$ is preferred to $y$, but the winning probability $\pi$ is determined by a random device such that with probability $\alpha_{i}$ the value of $\pi$ is $\pi_{i}$. We denote such lotteries as $\left\langle\pi_{1}, \alpha_{1} ; \ldots ; \pi_{n}, \alpha_{n}\right\rangle$. This is a two-stage lottery, where in the first stage, with probability $\alpha_{i}$ the winning probability of the second stage is determined to be $\pi_{i}$. The second stage is a simple lottery over the final outcomes $x$ and $y$, where the former is obtained with probability $\pi_{i}$.

Consider first the TC mechanism. Since there is an excess supply of $g_{1}$ $(p>q)$, all those who receive $g_{2}$ - their proportion is $1-p$ of the population - know that they will end up with their desired outcome regardless of their preferences. Either they will like it and keep it, or they will be able to trade. The size of the group of those who will receive $g_{2}$ but would like to replace it with $g_{1}$ is $q(1-p)$.

Those who receive $g_{1}$ (proportion $p$ of the population) will not know their true status until their preferences and trading outcomes are revealed. With probability $q$ they will like their outcome, but with probability $1-q$ they will look for a trading partner. In the latter case, the conditional probability of successful trade is

$$
\frac{q(1-p)}{(1-q) p}
$$

Their overall probability of satisfaction is therefore

$$
q+(1-q) \frac{q(1-p)}{(1-q) p}=\frac{q}{p}<1
$$

The TC mechanism thus yields the lottery over probabilities of receiving the desired outcome which is given by $X_{1}=\left\langle 1,1-p ; \frac{q}{p}, p\right\rangle$. This is an ex-ante lottery - before individuals know the outcome of the allocation mechanism, and of course before they know their preferences and the outcome of the trading period.

Consider now the SD mechanism (still assuming $p>q$ ). Out of the first $\frac{1-p}{1-q}$ individuals, $(1-q) \times \frac{1-p}{1-q}=1-p$ will choose $g_{2}$ and $q \times \frac{1-p}{1-q}<p$ will choose $g_{1}$. As $g_{2}$ is exhausted by the first $\frac{1-p}{1-q}$ individuals, the other $\frac{p-q}{1-q}$ will be able 
to satisfy their desires only if they prefer $g_{1}$ to $g_{2}$. The probability of having these preferences is $q$. SD thus leads to the lottery over probabilities given by $X_{2}=\left\langle 1, \frac{1-p}{1-q} ; q, \frac{p-q}{1-q}\right\rangle$. Here too the analysis is ex-ante, before individuals know their position in the queue or their preferences (which will only be revealed to them later). Observe that if $p=q$, then in a large economy both TC and SD yield (almost) everyone his desired outcome for sure.

\section{Are TC and SD Equivalent?}

The two lotteries over the probabilities of success we have previously discussed, $X_{1}=\left\langle 1,1-p ; \frac{q}{p}, p\right\rangle$ and $X_{2}=\left\langle 1, \frac{1-p}{1-q} ; q, \frac{p-q}{1-q}\right\rangle$, have the same "expected value." That is, the expected probability of receiving the preferred good is the same under both mechanisms, which is $1-p+q$. This is not surprising. As $p$, the proportion of good $g_{1}$, is greater than $q$, the proportional demand for $g_{1}$, it must be that eventually $p-q$ agents will not be happy with their outcome. Ex-ante, when agents do not yet know their preferences and the outcome of the mechanism, the reduced probability of success for each of them is therefore $1-p+q$. This, however, does not necessarily mean that all mechanisms with this reduced probability are equally attractive.

Let $x=$ "receive the desired outcome" and $y=$ "receive the undesired outcome." As $x$ and $y$ are fixed, the probability $\pi_{i}$ represents the lottery $\left(x, \pi_{i} ; y, 1-\pi_{i}\right)$. The decision maker has preferences $\succeq$ over compound lotteries of the form $\left\langle\pi_{1}, \alpha_{1} ; \ldots ; \pi_{n}, \alpha_{n}\right\rangle$ which can be represented by a functional $V$. Following Kreps and Porteus (1978) and Segal (1990), we use the recursive analysis of preferences over compound lotteries, where the decision maker considers the two-stage lottery $\left\langle\pi_{1}, \alpha_{1} ; \ldots ; \pi_{n}, \alpha_{n}\right\rangle$ as a lottery over his subjective values of the lotteries $\left(x, \pi_{i} ; y, 1-\pi_{i}\right)$. In particular, we do not assume the reduction of compound lotteries axiom, hence $V$ is not ordinally equivalent to $\sum \alpha_{i} \pi_{i}$.

We analyze mechanisms as cumulative distribution functions over $[0,1]$, where $F_{X}\left(\pi^{*}\right)$ is the probability that the mechanism $X$ yields a simple lottery $(x, \pi ; y, 1-\pi)$ with $\pi \leqslant \pi^{*}$. The set of feasible mechanisms is a strict subset of all simple lotteries (that is, lotteries with finite support) over the interval $[0,1]$, whose outcomes are the winning probability $\pi$ of the lottery $(x, \pi ; y, 1-\pi)$. In particular, we confine our attention to mechanisms in which the induced allocation is ex-post efficient, in the sense that after all units are

allocated, there are no two agents who can benefit from an exchange between 
them, where at least one of these improvements is strict. Ex-post efficiency implies that every individual who prefers the good for which there is excess supply must obtain it, as otherwise there will be scope for an improving trade. Therefore, any lottery in which some fraction of the population know for sure that independent of their preferences they will not receive their desired outcome (that is, any lottery over ex-ante probabilities in which $\pi_{i}=0$ is in its support) will be inefficient and will not be considered a valid mechanism. On the other hand, both TC and SD are ex-post efficient. It is enough to show that there is no agent who holds the item for which there is excess demand while he prefers the other good. By the construction of the TC mechanism, any such individual will participate in the second stage trade. In $\mathrm{SD}$, such an individual will never choose this good when his turn arrives, as his preferred good, which is in excess supply, will be still available.

Following Machina (1982), we assume first that the representation function $V$ is smooth in the sense of being Fréchet differentiable: For every $F$ there exists a continuous local utility function $u_{F}(\cdot)$ over $[0,1]$ such that

$$
V(G)-V(F)=\int_{0}^{1} u_{F}(\pi) d(G(\pi)-F(\pi))+o(\|G-F\|)
$$

Fréchet differentiability implies that when individuals evaluate small changes from the distribution $F$, they act precisely as would an expected utility maximizer with (Bernoulli) utility function $u_{F}(\cdot)$. We maintain this assumption on preferences mostly for tractability and conciseness. In Section $\mathrm{V}$ we use the popular rank-dependent utility model to demonstrate that a similar analysis can be performed for other, non-Fréchet differentiable preferences.

For $\alpha \in[0,1]$, denote by $G_{\alpha}$ the distribution of

$$
\alpha X_{1}+(1-\alpha) X_{2}=\left\langle 1, \frac{(1-p)(1-\alpha q)}{1-q} ; \frac{q}{p}, \alpha p ; q, \frac{(1-\alpha)(p-q)}{1-q}\right\rangle
$$

Proposition 1 If for all $\alpha$ the local utility of $V$ at $G_{\alpha}$ is concave, then $X_{1} \succ X_{2}$ and TC is preferred to SD. If all these local utilities are convex, then $X_{2} \succ X_{1}$ and SD is preferred to TC.

Proof: Observe that for $p>q, X_{2}$ is a mean preserving spread of $X_{1}$, and therefore for $\alpha>\alpha^{\prime}, G_{\alpha^{\prime}}$ is a mean preserving spread of $G_{\alpha}$. The proposition now follows from Theorem 2 in Machina (1982). 
Risk aversion along a segment connecting two lotteries may be a reasonable assumption when lotteries are over monetary payoffs, but such an attitude is much less obvious in the present context. To illustrate, consider lotteries of the form $Y_{\varepsilon}=\left\langle\frac{1}{2}-\varepsilon, \frac{1}{2} ; \frac{1}{2}+\varepsilon, \frac{1}{2}\right\rangle$. Obviously they are ordered by mean-preserving spread, where for $\varepsilon^{\prime}>\varepsilon, Y_{\varepsilon^{\prime}}$ is a mean preserving spread of $Y_{\varepsilon}$. However, there is no obvious reason to posit a specific ranking between $Y_{0}$ and $Y_{0.5}$. If time is not involved, it seems plausible to assume that $Y_{0} \sim Y_{0.5}$, as both represent a simple even chance of winning. If the passage of real time is considered, then preferences between the two capture preferences over the timing of resolution of uncertainty, as $Y_{0.5}$ is fully resolved in the current period, whereas $Y_{0}$ only resolves later. As we will further discuss in Section IV, there is no empirically obvious pattern for such preferences. We are thus interested also in situations where the local utilities are not always concave or always convex. We use this in the next section where we offer a new mechanism and show conditions under which this mechanism is better than both TC and SD.

\section{The Priority Groups Mechanism}

In this section we offer an alternative new mechanism, called Priority Groups (PG), and provide conditions under which it is preferred to both TC and SD. This mechanism first allocates the two goods as in the TC mechanism, and determines the trading probabilities of the $(1-q) p$ group of people who will find out that they received $g_{1}$, the good they do not like but for which there is an excess supply.

The formal procedure is as follows. In the first stage, allocate the two goods at random where each person has probability $p$ of getting $g_{1}$ and $1-p$ of getting $g_{2}$. Also, select $n$ priority groups where each person has probability $r_{i}$ of being a member of group $i, i=1, \ldots, n$, where $\sum_{i} r_{i}=1$, and assign each group probability $s_{i}, 1=s_{1}>\ldots>s_{n}$, such that the overall probability for trade of the unlucky $(1-q) p$ group equals the sum of the subgroups' probabilities (see eq. (1)). That is,

$$
\sum_{i} r_{i} s_{i}=\frac{q(1-p)}{(1-q) p}
$$

Since there is an excess demand for $g_{2}$, those who get it are guaranteed to eventually obtain their desired outcome. The size of this set is $1-p$. Also, 
those who will be assigned to the top priority group will obtain their desired outcome even if they received $g_{1}$. The size of this set is $p r_{1}$. As the allocation of the goods and the assignments to the priority group are simultaneous, exante each person knows that with probability $1-p+p r_{1}$ his probability of getting his desired outcome is 1 .

Those who get $g_{1}$ and are assigned to group $i$ (the probability of this event is $p r_{i}$ ) know that either they will like it and keep it, or they won't like it and will be able to trade with probability $s_{i}$. That is, with probability $p r_{i}$ they have $q+(1-q) s_{i}$ probability of getting their desired outcome. The PG mechanism is therefore the following lottery over probabilities

$$
\left\langle 1,1-p+p r_{1} ; q+(1-q) s_{2}, p r_{2} ; \ldots ; q+(1-q) s_{n}, p r_{n}\right\rangle
$$

This mechanism is ex-post efficient. Those who like $g_{1}$ will not end up with $g_{2}$. Either they receive $g_{1}$ and keep it, or they receive $g_{2}$ but will be able to trade it. The next claim shows that the reduced probability of this mechanism is the same as the one we computed at the beginning of Section III.

Claim 1 The reduced probability of lottery (3) is $1-p+q$, independently of $n, r_{i}$, and $s_{i}, i=1, \ldots, n$.

Proof: The reduced probability is

$$
\begin{aligned}
& 1-p+p r_{1}+\sum_{i=2}^{n}\left(q+(1-q) s_{i}\right) p r_{i}= \\
& 1-p+\sum_{i=1}^{n}\left(q+(1-q) s_{i}\right) p r_{i}= \\
& 1-p+q p+(1-q) p \sum_{i=1}^{n} r_{i} s_{i}=1-p+q
\end{aligned}
$$

where the first equality follows by $s_{1}=1$ and the last one by eq. (2).

Below, we analyze a special case of this mechanism and show conditions under which it is superior to both SD and TC. Specifically, we consider the case where $n=2, r_{1} \leqslant[q(1-p)] /[(1-q) p]$, and by eq. (2),

$$
s_{2}=\frac{q(1-p)}{(1-q) p r_{2}}-\frac{r_{1}}{r_{2}}=\frac{q(1-p)}{(1-q) p\left(1-r_{1}\right)}-\frac{r_{1}}{1-r_{1}}
$$


Let

$$
A\left(r_{1}\right)=q+\frac{(1-p) q-p(1-q) r_{1}}{p\left(1-r_{1}\right)}
$$

Eq. (3) becomes

$$
X_{3}\left(r_{1}\right)=\left\langle 1,1-p+p r_{1} ; A\left(r_{1}\right), p\left(1-r_{1}\right)\right\rangle
$$

Observe that if $r_{1}=0$, then $X_{3}\left(r_{1}\right)$ reduces to $X_{1}=\left\langle 1,1-p ; \frac{q}{p}, p\right\rangle$, the lottery obtained by the TC mechanism. On the other hand, if $r_{1}=\frac{q(1-p)}{p(1-q)}$, then by eq. (4) $s_{2}=0$ and $X_{3}\left(r_{1}\right)$ reduces to $X_{2}=\left\langle 1, \frac{1-p}{1-q} ; q, \frac{p-q}{1-q}\right\rangle$, the lottery obtained by the SD mechanism.

We now show that under some simple conditions, neither TC nor SD are optimal. We will do this by showing that moving from either in the direction of the PG mechanism will make individuals better off ex-ante.

Denote by $u_{r_{1}}$ be the local utility $u_{X_{3}\left(r_{1}\right)}$ of $V$ at $X_{3}\left(r_{1}\right)$.

Proposition 2 If $u_{0}$ is convex on $\left[\frac{q}{p}, 1\right]$ and $u_{\frac{q(1-p)}{p(1-q)}}$ is concave on $[q, 1]$, then neither TC nor SD is optimal.

Proof: By Machina's (1982) analysis

$$
\begin{aligned}
\frac{\partial}{\partial r_{1}} V\left(X_{3}\left(r_{1}\right)\right) & =\frac{\partial}{\partial r_{1}} \mathrm{E}\left[u_{r_{1}}\left(X_{3}\left(r_{1}\right)\right]\right. \\
& =p u_{r_{1}}(1)-\frac{p-q}{1-r_{1}} u_{r_{1}}^{\prime}\left(A\left(r_{1}\right)\right)-p u_{r_{1}}\left(A\left(r_{1}\right)\right)
\end{aligned}
$$

As $A(0)=\frac{q}{p}$, we get

$$
\left.\frac{\partial}{\partial r_{1}} V\left(X_{3}\left(r_{1}\right)\right)\right|_{r_{1}=0}=p u_{0}(1)-(p-q) u_{0}^{\prime}\left(\frac{q}{p}\right)-p u_{0}\left(\frac{q}{p}\right)
$$

which is positive if and only if

$$
\frac{p}{p-q}\left[u_{0}(1)-u_{0}\left(\frac{q}{p}\right)\right]>u_{0}^{\prime}\left(\frac{q}{p}\right)
$$

This inequality is satisfied whenever $u_{0}$ is convex on $\left[\frac{q}{p}, 1\right]$, hence TC is not optimal. 
For the second part, denote $r_{1}^{*}=\frac{q(1-p)}{p(1-q)}$. As $A\left(r_{1}^{*}\right)=q$, we get

$$
\left.\frac{\partial}{\partial r_{1}} V\left(X_{3}\left(r_{1}\right)\right)\right|_{r_{1}=r_{1}^{*}}=p u_{r_{1}^{*}}(1)-p(1-q) u_{r_{1}^{*}}^{\prime}(q)-p u_{r_{1}^{*}}(q)
$$

which is negative if and only if

$$
\frac{1}{(1-q)}\left[u_{r_{1}^{*}}(1)-u_{r_{1}^{*}}(q)\right]<u_{r_{1}^{*}}^{\prime}(q)
$$

This inequality is satisfied whenever $u_{r_{1}^{*}}$ is concave on $[q, 1]$, hence SD is not optimal.

Note that the functions $u_{0}$ and $u_{r_{1}^{*}}$ are local utilities at two different distributions, and therefore do not restrict each other.

As we have pointed out in the previous section, the conditions in Proposition 2 are not implausible. Theoretically, they are tied to forms of intrinsic preferences towards the timing of resolution of uncertainty. ${ }^{3}$ Recent experimental studies (for example, Ahlbrecht and Weber (1997), Kocher, Krawczyk, and van Winden (2014), Masatlioglu, Orhun, and Raymond (2017), and Nielsen (2020); see also Dillenberger and Segal (2017) and references therein) suggest that there is no conclusive evidence about which attitudes towards the resolution of uncertainty is the most prominent. These change based on the nature of the outcomes (goods or bads), the framing of the problem (choosing among information structures or among compound lotteries), and the skewness (positive or negative) of the underlying distribution over the probabilities. Furthermore, not only is it unclear which type is most prominent in a heterogeneous population, but also whether we can characterize an individual as having a global attitude in this regard. The latter is reflected in different curvatures of the local utility at different points.

\section{Rank-Dependent Utility}

Thus far we have confined attention to functionals $V$ that are Fréchet differentiable. While many known models are consistent with this assumption (for

\footnotetext{
${ }^{3}$ Kreps and Porteus (1978) pointed out that in a recursive setting, risk aggregation (about future consumption) takes place in the period in which it is resolved. They establish a link, within the recursive expected utility model, between the curvature of the utility functions in different periods and preferences over the timing of resolution of uncertainty.
} 
example, quadratic utility (Chew, Epstein, and Segal (1991)) and weighted expected utility (Chew (1983))), there are many popular models that are not. A prominent example is rank-dependent utility (Quiggin (1982)). ${ }^{4}$ Our aim is to show that the possible improvement of the PG mechanism over both TC and SD holds more generally. We demonstrate this by providing sufficient conditions on a version of the rank-dependent utility functional known as the dual theory (Yaari (1987)). ${ }^{5}$

Since $1>A\left(r_{1}\right)$, the rank-dependent value of $X_{3}\left(r_{1}\right)$ (see eq. (5)) is

$$
V_{\mathrm{RD}}\left(X_{3}\left(r_{1}\right)\right)=u\left(A\left(r_{1}\right)\right) g\left(p\left(1-r_{1}\right)\right)+u(1)\left[1-g\left(p\left(1-r_{1}\right)\right)\right]
$$

By the definition of $A\left(r_{1}\right)$ we get

$$
\begin{aligned}
\frac{\partial}{\partial r_{1}} V_{\mathrm{RD}}\left(X_{3}\left(r_{1}\right)\right)= & \frac{\partial}{\partial r_{1}}\left[u\left(A\left(r_{1}\right)\right) g\left(p\left(1-r_{1}\right)\right)+u(1)\left[1-g\left(p\left(1-r_{1}\right)\right)\right]\right]= \\
& -\left(\frac{p-q}{p\left(1-r_{1}\right)^{2}}\right) u^{\prime}\left(A\left(r_{1}\right)\right) g\left(p\left(1-r_{1}\right)\right) \\
& -p u\left(A\left(r_{1}\right)\right) g^{\prime}\left(p\left(1-r_{1}\right)\right)+p u(1) g^{\prime}\left(p\left(1-r_{1}\right)\right)
\end{aligned}
$$

Recall that $r_{1}=0$ represents the TC case. We get

$$
\left.\frac{\partial}{\partial r_{1}} V_{\mathrm{RD}}\left(X_{3}\left(r_{1}\right)\right)\right|_{r_{1}=0}=-\frac{p-q}{p} u^{\prime}\left(\frac{q}{p}\right) g(p)-p g^{\prime}(p)\left[u\left(\frac{q}{p}\right)-u(1)\right]
$$

For example, for $u(\pi)=\pi$ this equation becomes

$$
-\frac{p-q}{p} g(p)-p\left[\frac{q-p}{p}\right] g^{\prime}(p)
$$

which is positive if and only if

$(*) \eta_{g}(p)>1$

${ }^{4}$ Other examples include Gul (1991) and Cerreia-Vioglio, Dillenberger, and Ortoleva (2015).

${ }^{5}$ If we order the prizes in the support of a lottery $\left\langle\pi_{1}, \alpha_{1} ; \ldots ; \pi_{n}, \alpha_{n}\right\rangle$, with $\pi_{1}>\pi_{2}>$ $\ldots>\pi_{n}$, then the functional form for rank-dependent utility is: $V\left(\left\langle\pi_{1}, \alpha_{1} ; \ldots ; \pi_{n}, \alpha_{n}\right\rangle\right)=$ $u\left(\pi_{n}\right) g\left(\alpha_{n}\right)+\sum_{i=1}^{n-1} u\left(\pi_{i}\right)\left[g\left(\sum_{j=i}^{n} \alpha_{j}\right)-g\left(\sum_{j=i+1}^{n} \alpha_{j}\right)\right]$, where $g:[0,1] \rightarrow[0,1]$ is strictly increasing and onto, and $u:[0,1] \rightarrow \mathbb{R}$ is increasing. In the dual theory, $u$ is the identity function. We will assume in this section that $g$ is differentiable. 
The SD case is obtained when $r_{1}=\frac{q(1-p)}{p(1-q)}$. Now

$$
\begin{aligned}
& \left.\frac{\partial}{\partial r_{1}} V_{\mathrm{RD}}\left(X_{3}\left(r_{1}\right)\right)\right|_{r_{1}=\frac{q(1-p)}{p(1-q)}}= \\
& -\frac{p(1-q)^{2}}{p-q} u^{\prime}(q) g\left(\frac{p-q}{1-q}\right)-p g^{\prime}\left(\frac{p-q}{1-q}\right)[u(q)-u(1)]
\end{aligned}
$$

which is negative if and only if

$$
\frac{u^{\prime}(q)(1-q)}{1-u(q)}>\eta_{g}\left(\frac{p-q}{1-q}\right)
$$

For $u(\pi)=\pi$ this condition becomes

$(* *) 1>\eta_{g}\left(\frac{p-q}{1-q}\right)$

It is common to assume that $g$ is an inverse S-shaped function - concave for small probabilities and convex for high probabilities. ${ }^{6}$ This property captures a tendency to overweight both best and worst events that occur with small probabilities. The two conditions, $(*)$ and $(* *)$, are consistent with this shape of $g$. It is indeed easy to construct functional forms for $g$ that satisfy both.

The two conditions $(*)$ and $(* *)$ are sufficient to show that there are PG mechanisms with two groups that are better than both TC and SD. A natural question is whether there is an optimal PG mechanism, at least for the case $n=2$ and $u(\pi)=\pi$. Since the rank-dependent value of $X_{3}\left(r_{1}\right)$ is a continuous function on a closed segment, then, by eq. (6), a necessary condition for an interior optimum is

$$
\begin{gathered}
-\left(\frac{p-q}{p\left(1-r_{1}\right)^{2}}\right) g\left(p\left(1-r_{1}\right)\right)+p\left[1-A\left(r_{1}\right)\right] g^{\prime}\left(p\left(1-r_{1}\right)\right)=0 \\
\Longrightarrow\left(\frac{1}{p\left(1-r_{1}\right)}\right) g\left(p\left(1-r_{1}\right)\right)=g^{\prime}\left(p\left(1-r_{1}\right)\right) \Longrightarrow \eta_{g}\left(p\left(1-r_{1}\right)\right)=1
\end{gathered}
$$

\footnotetext{
${ }^{6}$ For empirical evidence in favor of inverse S-shaped probability weighting, see, among others, Gonzales and Wu (1989), Tversky and Kahneman (1992), and Fehr-Duda and Epper (2012).
} 
If $\eta_{g}$ is strictly increasing, there is a unique $r_{1}^{*}$ with this property. Note that this condition is consistent with $(*)$ and $(* *)$ since $p>p(1-r)>\frac{p-q}{1-q}$ (recall that $r_{1} \in\left[0, \frac{q(1-p)}{p(1-q)}\right]$ ). Differentiate again to obtain

$$
-\frac{p-q}{1-r_{1}}\left[\frac{2 g\left(p\left(1-r_{1}\right)\right)}{p\left(1-r_{1}\right)^{2}}-\frac{2 g^{\prime}\left(p\left(1-r_{1}\right)\right)}{1-r_{1}}+g^{\prime \prime}\left(p\left(1-r_{1}\right)\right)\right]
$$

Using eq. (7), the sign of expression (8) is the same as the sign of $-g^{\prime \prime}(p(1-$ $\left.\left.r_{1}^{*}\right)\right)$. It is thus negative if $g^{\prime \prime}\left(p\left(1-r_{1}^{*}\right)\right)>0$, in which case $r_{1}^{*}$ is indeed optimal.

\section{Known Preferences}

In previous sections we studied the ex-ante case, where the first part of the mechanism is implemented before individuals know their own preferences. A similar method can be used to study the case where individuals know their preferences from the beginning. Here we demonstrate how our analysis can be easily applied to this case as well. As before, we confine attention to the case of large (continuum) economies and assume, without loss of generality, that there is an excess supply of $g_{1}$, that is, $p>q$. In the TC mechanism we can therefore identify four groups:

1. $q p$ will get $g_{1}$ and like it.

2. $(1-q) p$ will get $g_{1}$ and will prefer to trade it for $g_{2}$.

3. $q(1-p)$ will get $g_{2}$ and will prefer to trade it for $g_{1}$.

4. $(1-q)(1-p)$ will get $g_{2}$ and like it.

Since $p>q$, the third group is smaller than the second one, and therefore all members of the third group will be able to trade. In other words, all those who prefer $g_{1}$ (the first and the third group) are guaranteed to receive it. Those who prefer $g_{2}$ face a lottery. With probability $1-p$ they will get their desired outcome, and with probability $p$ they will get their desired outcome if they will be able to trade, the probability of this event is $\frac{q(1-p)}{(1-q) p}$. Their underlying conditional lottery is thus $W_{1}=\left\langle 1,1-p ; \frac{q(1-p)}{(1-q) p}, p\right\rangle$.

In the SD mechanism, the $q$ who prefer $g_{1}$ are guaranteed to receive it. A person who prefers $g_{2}$ will get it only if he is in the top $\alpha$ of the list 
where $(1-q) \alpha=1-p$, that is, if his rank is less than $\frac{1-p}{1-q}$. The underlying conditional lottery is thus $W_{2}=\left\langle 1, \frac{1-p}{1-q} ; 0, \frac{p-q}{1-q}\right\rangle$. Note that here, after the first step of the mechanism, all participants know for sure whether they will receive their preferred outcome or not.

We obtain that all those who prefer $g_{1}$ to $g_{2}$ ( $q$ of the group) know in advance that since there is an excess supply of their desired good, they will eventually get it under both procedures, and are therefore indifferent between the two mechanisms. Those who prefer $g_{2}$ to $g_{1}$ have to compare $W_{1}$ with $W_{2}$. Here again both mechanisms induce the same overall probability of success, $\frac{1-p}{1-q}$, while $W_{2}$ is a mean preserving spread of $W_{1}$, so that the same qualitative results as in Proposition 1 hold. The results from the rank-dependent utility model can be extended to this case as well.

The analysis of the PG mechanism is also analogous to the ex-ante case. It is now applied only for individuals who want $g_{2}$ (the others will get their desired outcome whether they initially hold it or trade for it.) As before, we start by randomly assigning the goods and by splitting individuals into two priority groups. Those who belong to the first group will be able to trade for sure. The probability of belonging to this group is $r_{1}$. With probability $1-r_{1}$, people belong to the second priority group and will be able to trade with probability $s_{2}$. To equate supply and demand, the constraint on $s_{2}$ is

$$
q(1-p)=(1-q) p\left(r_{1}+\left(1-r_{1}\right) s_{2}\right) \Longrightarrow s_{2}=\frac{q(1-p)-(1-q) p r_{1}}{(1-q) p\left(1-r_{1}\right)}
$$

Each of the individuals who want $g_{2}$ thus faces the following lottery

$$
W_{3}\left(r_{1}\right)=\left\langle 1,1-p+p r_{1} ; \frac{q(1-p)-(1-q) p r_{1}}{(1-q) p\left(1-r_{1}\right)}, p\left(1-r_{1}\right)\right\rangle
$$

where the first entry means that the desired outcome is guaranteed to those who hold $g_{2}$ or those who hold $g_{1}$ but are in the first priority group. Observe that if $r_{1}=0$ this reduces to TC, whereas SD corresponds to $r_{1}=\frac{q(1-p)}{(1-q) p}<1$.

Denote by $v_{r_{1}}$ be the local utility $v_{W_{3}\left(r_{1}\right)}$ of $V$ at $W_{3}\left(r_{1}\right)$. Mimicking the calculations as in the proof of Proposition 2 for $W_{3}\left(r_{1}\right)$ rather than $X_{3}\left(r_{1}\right)$, one obtains the following result.

Proposition 3 If $v_{0}$ is convex on $\left[\frac{q(1-p)}{(1-q) p}, 1\right]$ and $v_{\frac{q(1-p)}{(1-q) p}}$ is concave on $[0,1]$, then neither TC nor SD is optimal. 
Proof: By Machina's (1982) analysis

$$
\begin{aligned}
\frac{\partial}{\partial r_{1}} V\left(W_{3}\left(r_{1}\right)\right) & =\frac{\partial}{\partial r_{1}} \mathrm{E}\left[v_{r_{1}}\left(W_{3}\left(r_{1}\right)\right]\right. \\
& =p v_{r_{1}}(1)-\frac{p-q}{(1-q)\left(1-r_{1}\right)} v_{r_{1}}^{\prime}\left(\frac{A\left(r_{1}\right)-q}{1-q}\right)-p v_{r_{1}}\left(\frac{A\left(r_{1}\right)-q}{1-q}\right)
\end{aligned}
$$

As $A(0)=\frac{q}{p}$, we get

$$
\left.\frac{\partial}{\partial r_{1}} V\left(W_{3}\left(r_{1}\right)\right)\right|_{r_{1}=0}=p v_{0}(1)-\frac{p-q}{1-q} v_{0}^{\prime}\left(\frac{q(1-p)}{p(1-q)}\right)-p v_{0}\left(\frac{q(1-p)}{p(1-q)}\right)
$$

which is positive if and only if

$$
\frac{p(1-q)}{p-q}\left[v_{0}(1)-v_{0}\left(\frac{q(1-p)}{p(1-q)}\right)\right]>v_{0}^{\prime}\left(\frac{q(1-p)}{p(1-q)}\right)
$$

This inequality is satisfied whenever $u_{0}$ is convex on $\left[\frac{q(1-p)}{p(1-q)}, 1\right]$, hence TC is not optimal.

For the second part, denote $r_{1}^{*}=\frac{q(1-p)}{p(1-q)}$. As $A\left(r_{1}^{*}\right)=q$, we get

$$
\left.\frac{\partial}{\partial r_{1}} V\left(W_{3}\left(r_{1}\right)\right)\right|_{r_{1}=r_{1}^{*}}=p v_{r_{1}^{*}}(1)-p v_{r_{1}^{*}}^{\prime}(0)-p v_{r_{1}^{*}}(0)
$$

which is negative if and only if

$$
v_{r_{1}^{*}}(1)-v_{r_{1}^{*}}(0)<v_{r_{1}^{*}}^{\prime}(0)
$$

This inequality is satisfied whenever $v_{r_{1}^{*}}$ is concave on $[0,1]$, hence $\mathrm{SD}$ is not optimal.

\section{Discussion}

There is a vast literature on allocation problems of indivisible goods. This literature typically distinguishes between possible mechanisms based on criteria that are linked to the strategic interaction between the receiving agents and the agents' incentives to truthfully reveal any private information they may have. In this paper we take a different approach. We purposely abstract 
away from any strategic considerations by confining attention to a setting in which there are only two types of goods, and use individual preferences over mechanisms to compare them. We thus concentrate on the decision-theoretic dimension of the mechanisms without having to worry about individuals' manipulations of their preferences over the outcomes.

Random allocation mechanisms typically involve multi-stage lotteries. Based on a compelling evidence that people do not routinely use the laws of probability to reduce multi-stage lotteries, we postulate that individuals perceive mechanisms as compound lotteries and have recursive preferences over them. Simple and familiar conditions then allow us to compare mechanisms that are deemed identical in standard models. Moreover, our approach permits us to offer a new mechanism that under some conditions outperforms standard mechanisms.

In this paper we show that it is enough to have $n=2$ priority groups to (sometimes) improve upon both TC and SD. In the special case of rankdependent utility we also outline conditions for an optimal PG mechanism with two groups. We do leave open, however, the question of what is the optimal number of groups (together with the probability of trade assigned to each of them). This question crucially depends on the individuals' preferences.

There are some considerations about the actual implementation of the PG mechanism which we do not explicitly address in the paper. First, it is often the case that having more groups entails higher bureaucratic costs that may offset the benefit of having finer division of the population. Therefore, even if it is theoretically beneficial to have more priority groups, a cost-benefit analysis may dictate a smaller number of such groups.

Implementation of the extreme cases of TC and SD requires no knowledge of individual preferences or even aggregate preferences. The goods are allocated at random (TC) and individuals are ordered at random (SD) regardless of preferences. In order to determine which is better, however, society needs to have information about the value of $q$ and about individual preferences over lotteries.

The allocation of the goods in the PG mechanism does not require knowledge about $q$, but the determination of the sizes of the groups and their probabilities of trade needs to satisfy eq. (2) which requires $q .^{7}$ Moreover, as is

\footnotetext{
${ }^{7}$ For the analysis in Section V, knowing $q$ is needed only to the extent that it determines the possible range of $r_{1}$, the size of the group that is guaranteed the option to trade.
} 
demonstrated in Section $\mathrm{V}$, the optimal division into priority groups requires knowledge about individual preferences.

\section{References}

[1] Ahlbrecht, Martin, and Martin Weber. 1997. "Preference for gradual resolution of uncertainty," Theory and Decision 43:167-185.

[2] Abdellaoui, Mohammed, Peter Klibanoff, and Laetitia Placido. 2013. "Experiments on compound risk in relation to simple risk and to ambiguity," Management Science 61:1306-1322.

[3] Abdulkadiroğlu, Atila, and Tayfun Sönmez. 1998. "Random serial dictatorship and the core from random endowments in house allocation problems," Econometrica 66:689-701.

[4] Abdulkadiroğlu, Atila, and Tayfun Sönmez. 2013. "Matching markets: Theory and practice," Advances in Economics and Econometrics, D. Acemoglu, M. Arello, and E. Dekel (eds), Cambridge: CUP.

[5] Cerreia-Vioglio, Simone, David Dillenberger, and Pietro Ortoleva. 2015. "Cautious expected utility and the certainty effect," Econometrica 83:693-728.

[6] Chew, Soo Hong. 1983. "A generalization of the quasilinear mean with applications to the measurement of income inequality and decision theory resolving the Allais paradox," Econometrica 51:1065-1092.

[7] Chew, Soo Hong, Larry G. Epstein, and Uzi Segal. 1991. "Mixture symmetry and quadratic utility," Econometrica 59:139-163.

[8] Dillenberger, David, and Uzi Segal. 2017. "Skewed noise," Journal of Economic Theory 169:344-364.

[9] Fehr-Duda, Helga, and Thomas Epper. 2012. "Probability and risk: Foundations and economic implications of probability-dependent risk preferences," Annual Review of Economics 4:567-593.

[10] Gonzalez, Richard, and George Wu. 1999. "On the shape of the probability weighting function," Cognitive Psychology 38:129-166 
[11] Gul, Faruk. 1991. "A theory of disappointment aversion," Econometrica 59:667-686.

[12] Halevy, Yoram. 2007. "Ellsberg revisited: An experimental study," Econometrica 75:503-536.

[13] Harrison, Glen W., Jimmy Martinez-Correa, and J.Todd Swarthout. 2015. "Reduction of compound lotteries with objective probabilities: Theory and evidence," Journal of Economic Behavior and Organization $119(\mathrm{C}): 32-55$.

[14] Hylland, Aanund, and Richard Zeckhauser. 1979. "The efficient allocation of individuals to positions," Journal of Political Economy 87:293314 .

[15] Kocher, Martin G., Michal Krawczyk, and Frans van Winden. 2014. "Let me dream on! Anticipatory emotions and preference for timing in lotteries," Journal of Economic Behavior and Organization 98:29-40.

[16] Kreps, David M., and Evan L. Porteus. 1978. "Temporal resolution of uncertainty and dynamic choice theory," Econometrica 46:185-200.

[17] Machina, Mark J.. 1982. “'Expected utility' analysis without the independence axiom," Econometrica 50:277-323.

[18] Masatlioglu, Yusufcan, Yesim Orhun, and Collin Raymond. 2017. "Skewness and intrinsic preferences for information," Mimeo.

[19] Nielsen, Kirby. 2020. "Preferences for the resolution of uncertainty and the timing of information," Journal of Economic Theory 189: Article 105090 .

[20] Pathak, Parag A., and Jay Sethuraman. 2011. "Lotteries in student assignment: An equivalence result," Theoretical Economics 6:1-17.

[21] Quiggin, John. 1982. "A theory of anticipated utility," Journal of Economic Behavior and Organization 3:323-343.

[22] Segal, Uzi. 1990. "Two-stage lotteries without the reduction axiom," Econometrica 58:349-377. 
[23] Tversky, Amos, and Daniel Kahneman. 1992. "Advances in prospect theory: Cumulative representation of uncertainty," Journal of Risk and Uncertainty 5:297-323.

[24] Yaari, Menahem. 1987. "The dual theory of choice under risk," Econometrica 55:95-115. 